\title{
281
}

\section{The Percentile Points of Distributions Having Known Cumulants}

\author{
Sir Ronald A. FisheR \\ AND \\ E. A. Cornish
}

In an earlier study of the uses of moments and cumulants in the specification of statistical distributions, the authors developed explicit asymptotic expansions, expressing any desired percentile point of such distributions in terms of known cumulants.

The general formulae are now presented $d \mathrm{~s}$ far as the sixth adjustment, based on the eighth cumulant, and also numerical tables showing the coefficients of all terms for ten chosen levels of significance over the range 0.5 to 0.0005 (single tail), together with the first five Hermite polynomials and tables for the common tests of significance, $x^{2}, t$ and $z$, at the same levels.

\section{INTRODUCTORY}

In 1937, in a study of the uses of moments and cumulants in the specification of statistical distributions, the authors (1937)* were led to develop explicit asymptotic expansions, expressing any desired percentile point of such distributions in terms of known cumulants. The general formulae were presented so far as the fourth adjustment, based on the sixth cumulant, and also numerical tables showing the coefficients of all terms for nine chosen levels of significance over the range 0.25 to 0.0005 (single tail), together with the first five Hermite polynomials at the same levels.

As an illustrative example, the cumulants of the $z$ distribution were expressed in terms of $1 / n_{1}$ and $1 / n_{2}$, the reciprocals of the two numbers of degrees of freedom, and the rapid convergence at the $5 \%$ point exhibited for the case $n_{1}=24$ and $n_{2}=60$. In the intervening period, the formulae have frequently been found useful, either for calculations of higher accuracy in the case of functions already tabulated, or for values outside their range, or especially with tables of multiple entry to supply intermediate values more accurate than can be obtained by interpolation (Fisher 1941, Goldberg and Levine 1946), or for cases where no tables existed (Johnson and Welch 1939).

\section{The Method of Expansion}

The several steps in the method of expansion are set out in Sections 7 and 8 of our previous paper. Here a brief outline of these steps will be sufficient, as the principal purpose of this note is to extend the formulae and tables to the sixth corrective term, so widening the range of useful application.

If the element of frequency in the distribution of a variate $\xi$ is $f(\xi) d \xi$, the

\footnotetext{
*Attention is drawn to this reference, as it has been repeatedly misquoted.
} 
efect of the operator

$$
\exp \left\{\frac{a_{r}}{r !}\left(-\frac{d}{d \xi}\right)^{r}\right\}
$$

when acting on the frequency function, is to increase the $r$ th cumulant by $a_{r}$, but leaves the distribution otherwise unchanged. This important operational property of the cumulants is basic to the method, the essential steps of which are as follows:

(i) If the cumulants $\kappa_{1}, \kappa_{2}, \cdots$ of the distribution of $\xi$ are expressible in power series of the reciprocal of some number $n$, the frequency element may be represented as

$$
\begin{aligned}
\exp \left\{-a v^{1 / 2} \frac{d}{d \xi}+\frac{1}{2} b v \frac{d^{2}}{d \xi^{2}}-\frac{1}{6} c v^{3 / 2} \frac{d^{3}}{d \xi^{3}}+\frac{1}{24} d v^{2} \frac{d^{4}}{d \xi^{4}}-\frac{1}{120} e v^{5 / 2} \frac{d^{5}}{d \xi^{5}}\right. \\
\left.+\frac{1}{720} f v^{3} \frac{d^{8}}{d \xi^{6}}-\cdots\right\} \frac{1}{\sqrt{2 \pi v}} e^{-(\xi-m) \cdot / 2 v} d \xi
\end{aligned}
$$

where the coefficients $a$ and $c$ are of order $n^{-1 / 2}, b$ and $d$ of order $n^{-1}, e$ of order $n^{-3 / 2}, f$ of order $n^{-2} \cdots$, and are related, respectively, to $\kappa_{1}$ and $\kappa_{33}, \kappa_{2}$ and $\kappa_{4}, \kappa_{5}, \kappa_{B}, \cdots$, and $m$ and $v$ are the mean and variance of a normal distribution chosen for convenience.

(ii) Expanding the operator and integrating, the frequency less than $m+\xi v^{1 / 2}$ may be expressed in terms of the corresponding normal probability integral and a series of adjustments of decreasing order of magnitude.

(iii) If $x$ is the normal deviate at some chosen level of probability, and $\xi$ the corresponding deviate of the distribution under consideration, the difference $\xi-x$ may be found by equating the expression for the probability that the variate has a value less than $\xi$ to

$$
\begin{aligned}
\frac{1}{\sqrt{2 \pi}} \int_{-\infty}^{\xi} e^{-\xi^{1 / 2}} d \xi & -\frac{1}{\sqrt{2 \pi}} e^{-\xi^{2} / 2}\left\{(\xi-x)-\frac{1}{2}(\xi-x)^{2} \xi\right. \\
& \left.+\frac{1}{6}(\xi-x)^{3}\left(\xi^{2}-1\right)-\frac{1}{24}(\xi-x)^{4}\left(\xi^{3}-3 \xi\right)+\cdots\right\}
\end{aligned}
$$

in which the coefficients are the Hermite polynomials. By considering the terms of each order of magnitude in succession, we may develop an expansion for $\xi-x$ in terms of successive polynomials in $\xi$.

(iv) The expansion for $\xi-x$ is converted to a much more useful expansion in terms of $x$, the values of which are known in advance, so obtaining the percentile deviate $\xi$ explicitly in terms of the normal deviate.

The adjustments to the normal deviate having the required probability integral are set out in tabular form below. Adjustments $V$ and VI are new with this paper.

Table I gives the numerical values of the first seven Hermite polynomials, over the same range of percentiles as given previously, and Table II gives the numerical values of the polynomials in the several adjustment terms. 


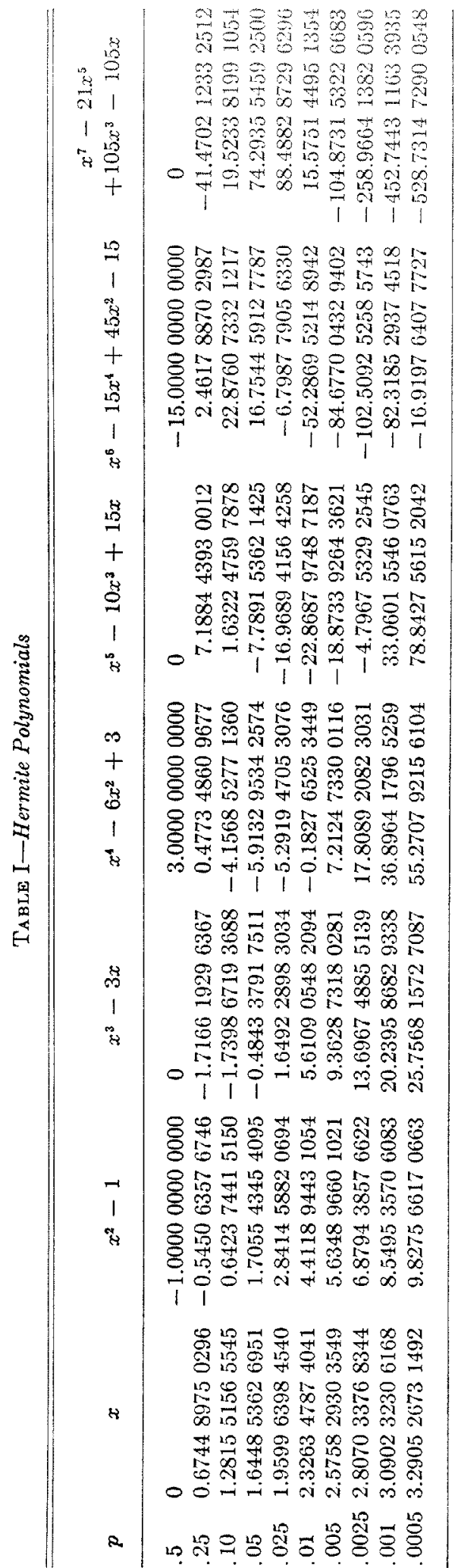




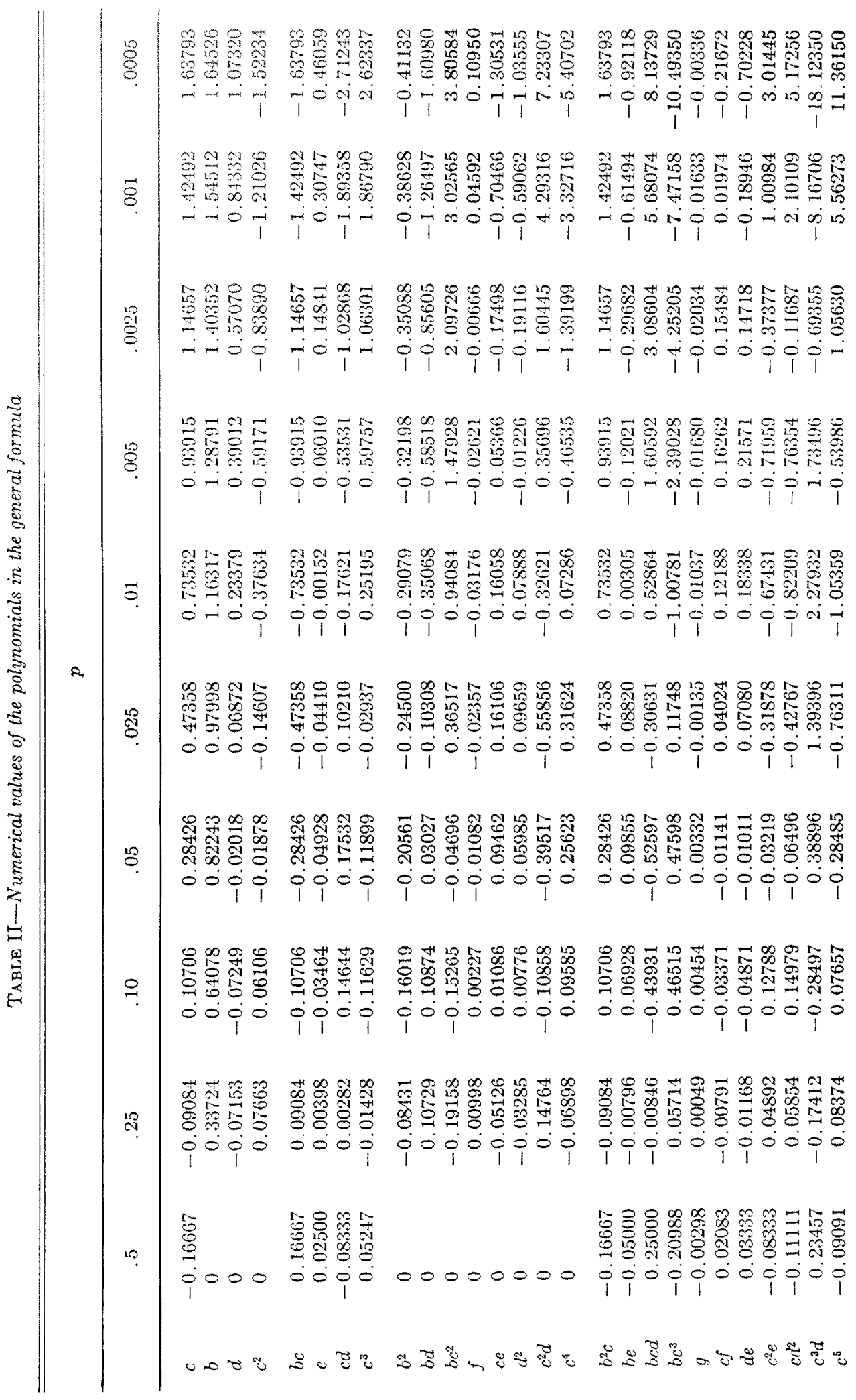




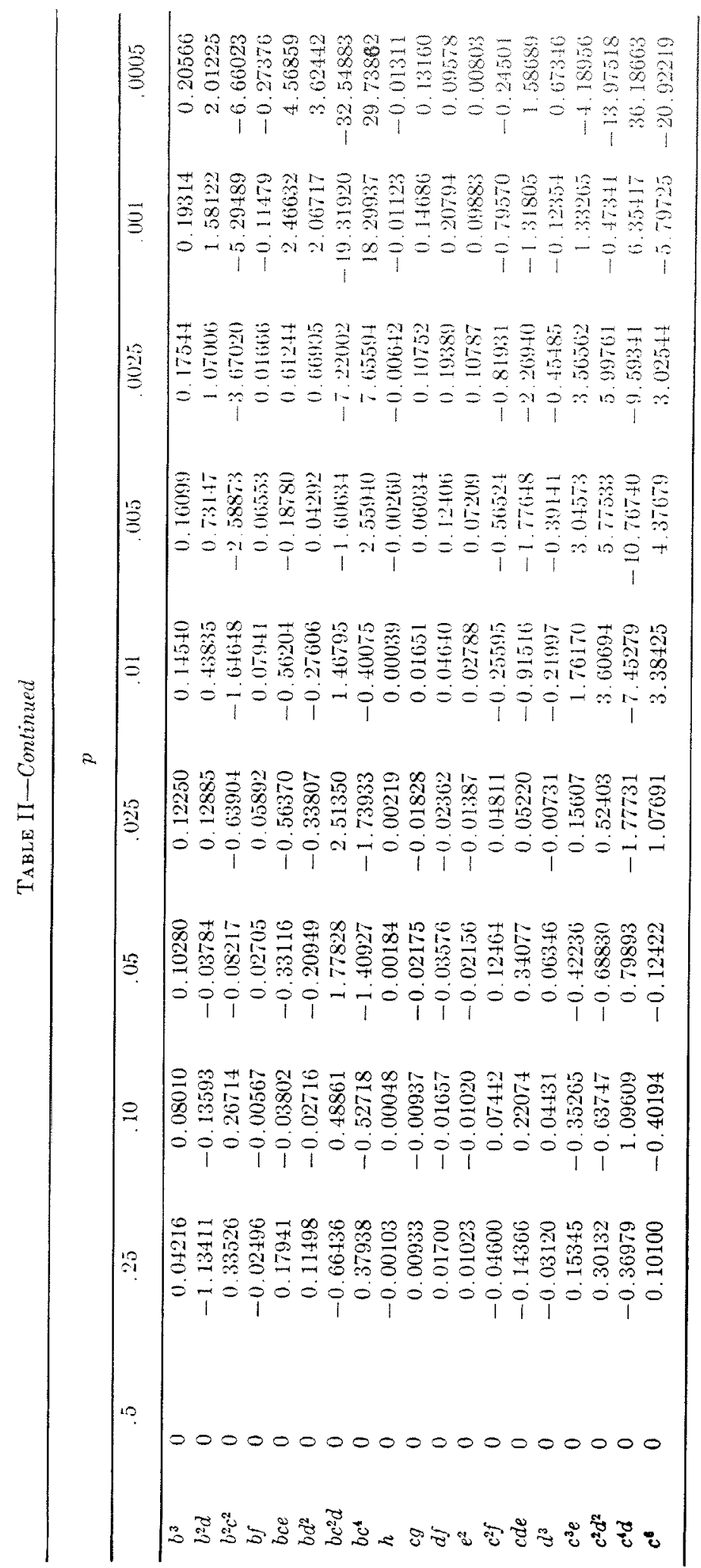


Adjust-

ment Coefficient

\begin{tabular}{llr}
\hline VI & $x$ & 16 \\
$b^{2} d$ & $5\left(x^{3}-3 x\right)$ & 64 \\
$b^{2} c^{2}$ & $-35\left(2 x^{3}-5 x\right)$ & 288 \\
$b f$ & $-\left(x^{5}-10 x^{3}+15 x\right)$ & 288 \\
$b c e$ & $7\left(2 x^{5}-17 x^{3}+21 x\right)$ & 360 \\
$b d^{2}$ & $7\left(3 x^{5}-24 x^{3}+29 x\right)$ & 768 \\
$b c^{2} d$ & $-\left(14 x^{5}-103 x^{3}+107 x\right)$ & 64 \\
$l c^{4}$ & $11\left(252 x^{5}-1688 x^{3}+1511 x\right)$ & 15552 \\
$h$ & $x^{7}-21 x^{5}+105 x^{3}-105 x$ & 40320 \\
$c g$ & $-\left(2 x^{7}-37 x^{5}+160 x^{3}-135 x\right)$ & 5040 \\
$d f$ & $-\left(x^{7}-17 x^{5}+69 x^{3}-57 x\right)$ & 1152 \\
$e^{2}$ & $-\left(2 x^{7}-33 x^{5}+132 x^{3}-108 x\right)$ & 3600 \\
$c^{2} f$ & $18 x^{7}-293 x^{5}+1100 x^{3}-795 x$ & 5184 \\
$c d e$ & $18 x^{7}-273 x^{5}+974 x^{3}-695 x$ & 1440 \\
$d^{3}$ & $9 x^{7}-131 x^{3}+451 x^{3}-321 x$ & 3072 \\
$c^{3} e$ & $-\left(396 x^{7}-5708 x^{3}+18755 x^{3}-11811 x\right)$ & 19440 \\
$c^{2} d^{2}$ & $-\left(594 x^{7}-8193 x^{5}+26006 x^{3}-16367 x\right)$ & 13824 \\
$c^{4} d$ & $5148 x^{7}-67004 x^{5}+195259 x^{3}-109553 x$ & 62208 \\
$c^{6}$ & $-\left(154440 x^{7}-1887684 x^{5}+5033714 x^{3}-2542637 x\right)$ & 4199040
\end{tabular}

(a) $\chi^{2}$ distribution

If $n$ is the number of degrees of freedom

$$
\begin{gathered}
\chi^{2}=n \\
+\sqrt{n}(x \sqrt{2}) \\
+\frac{2}{3}\left(x^{2}-1\right) \\
+\frac{1}{\sqrt{n}}\left(\frac{x^{3}-7 x}{9 \sqrt{2}}\right) \\
-\frac{1}{n}\left(\frac{6 x^{4}+14 x^{2}-32}{405}\right) \\
+\frac{1}{n \sqrt{n}}\left(\frac{9 x^{5}+256 x^{3}-433 x}{4860 \sqrt{2}}\right) \\
+\frac{1}{n^{2}}\left(\frac{12 x^{6}-243 x^{4}-923 x^{2}+1472}{25515}\right) \\
-\frac{1}{n^{2} \sqrt{n}}\left(\frac{3753 x^{7}+4353 x^{5}-289517 x^{3}-289717 x}{9185400 \sqrt{2}}\right) .
\end{gathered}
$$




\section{(b) taistribution}

If $n$ is the number of degrees of freedom

$$
\begin{gathered}
t=x \\
+\frac{1}{n}\left(\frac{x^{3}+x}{4}\right) \\
+\frac{1}{n^{2}}\left(\frac{5 x^{5}+16 x^{3}+3 x}{96}\right) \\
+\frac{1}{n^{3}}\left(\frac{3 x^{7}+19 x^{5}+17 x^{3}-15 x}{384}\right) \\
+\frac{1}{n^{4}}\left(\frac{79 x^{9}+776 x^{7}+1482 x^{5}-1920 x^{3}-945 x}{92160}\right) \\
+\frac{1}{n^{5}}\left(\frac{27 x^{11}+339 x^{0}+930 x^{7}-1782 x^{5}-765 x^{3}+17955 x}{368640}\right) .
\end{gathered}
$$

The number of terms and the orders of magnitude differ in this expansion because it has been derived from the original expansion of Student's integral in powers of $n^{-1}$ as given by Fisher (1926).

(c) z distribution

If $n_{1}$ and $n_{2}$ are the degrees of freedom, and

$$
\sigma=\frac{1}{n_{1}}+\frac{1}{n_{2}} \quad \delta=\frac{1}{n_{1}}-\frac{1}{n_{2}},
$$

then

$$
\begin{aligned}
& z=\sqrt{\frac{\sigma}{2}}(x) \\
& -\delta\left(\frac{x^{2}+2}{6}\right) \\
& +\sqrt{\frac{\sigma}{2}}\left\{\sigma\left(\frac{x^{3}+3 x}{24}\right)+\frac{\delta^{2}}{\sigma}\left(\frac{x^{3}+11 x}{72}\right)\right\} \\
& -\left\{\delta \sigma\left(\frac{x^{4}+9 x^{2}+8}{120}\right)-\frac{\delta^{3}}{\sigma}\left(\frac{3 x^{4}+7 x^{2}-16}{3240}\right)\right\} \\
& +\sqrt{\frac{\sigma}{2}}\left\{\sigma^{2}\left(\frac{x^{5}+20 x^{3}+15 x}{1920}\right)+\delta^{2}\left(\frac{x^{5}+44 x^{3}+183 x}{2880}\right)\right. \\
& \left.+\frac{\delta^{4}}{\sigma^{2}}\left(\frac{9 x^{5}-284 x^{3}-1513 x}{155520}\right)\right\}+\left\{\delta \sigma^{2}\left(\frac{4 x^{6}-25 x^{4}-177 x^{2}+192}{20160}\right)\right. \\
& \left.+\delta^{3}\left(\frac{4 x^{8}+101 x^{4}+117 x^{2}-480}{90720}\right)-\frac{\delta^{5}}{\sigma^{2}}\left(\frac{12 x^{6}+513 x^{4}+841 x^{2}-2560}{1632960}\right)\right\}
\end{aligned}
$$




$$
\begin{gathered}
\text { PERCENTIE POINTS OF DISTRIBUTONS } \\
-\sqrt{\frac{\sigma}{2}\left\{\sigma^{3}\left(\frac{x^{7}+7 x^{5}+7 x^{3}+105 x}{21501}\right)\right.} \\
+\delta \sigma^{2}\left(\frac{801 x^{7}+10511 x^{5}+30151 x^{3}+62241 x}{4838400}\right) \\
-\frac{\delta^{4}}{\sigma}\left(\frac{477 x^{7}+4507 x^{5}-82933 x^{3}-264363 x}{43545600}\right) \\
\left.+\frac{\delta^{6}}{\sigma^{3}}\left(\frac{3753 x^{7}+55383 x^{5}-368897 x^{3}-1213927 x}{1175731200}\right)\right\} .
\end{gathered}
$$

\begin{tabular}{|c|c|}
\hline \multicolumn{2}{|c|}{ Successive terms } \\
\hline - & 1960643 \\
\hline & 446851 \\
\hline - & 48004 \\
\hline - & 154 \\
\hline - & 102 \\
\hline
\end{tabular}

As an illustration of the accuracy, we may use the example given previously. When $n_{1}=24$ and $n_{2}=60$, the $5 \%$ value of $z$ is 0.26534844 , and the asymptotic expansion yields the following values:

Order of
magnitude
0
1
2
3
4
5
6

$\begin{array}{cc}\text { Successive totals } \\ 0.2809 & 1224 \\ 2613 & 0581 \\ 2657 & 7432 \\ 2652 & 9428 \\ 2653 & 5073 \\ 2653 & 4919 \\ 2653 & 4817\end{array}$

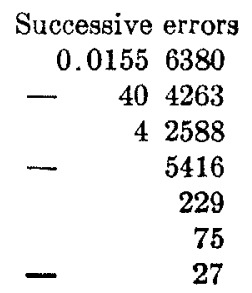

The numerical values of the polynomials in $x$ occurring in the above formulae are given in Table III.

\section{Examples of the Types of Problem to Which the Expansion Has Been Applied}

\section{(a) The asymptotic approach to Behrens' integral}

Fisher (1926) developed the ordinate and integral of Student's distribution in a series of powers of $n^{-1}$, giving the polynomial coefficients so far as the fifth adjustment. The purpose of this expansion was to supply sufficiently accurate values of the probabilities corresponding to any values of $t$ for values of $n$ beyond the range which it was proposed to tabulate.

With Behrens' extension of Student's test there were even stronger reasons for using a similar method. The direct calculations carried out by Sukhatme (1938) are very much more laborious than those needed for Student's integral. At any single level of significance, various values are required for three parameters, provided by the two numbers of degrees of freedom of the two samples, and the estimated ratio of the variances of the two means. For functions of many variables, there is a great advantage in the use of explicit formulae in which the several variables may be substituted, and there is much to be gained by extending the use of such formulae over regions too extensive for complete tabulation. Finally, it should be noted that the logical situation in which we would prefer to rely on the separate estimates of variances from the two samples rather than on any process of pooling these estimates, is of more frequent occurrence with large samples than with small, and is particularly applicable to cases, 


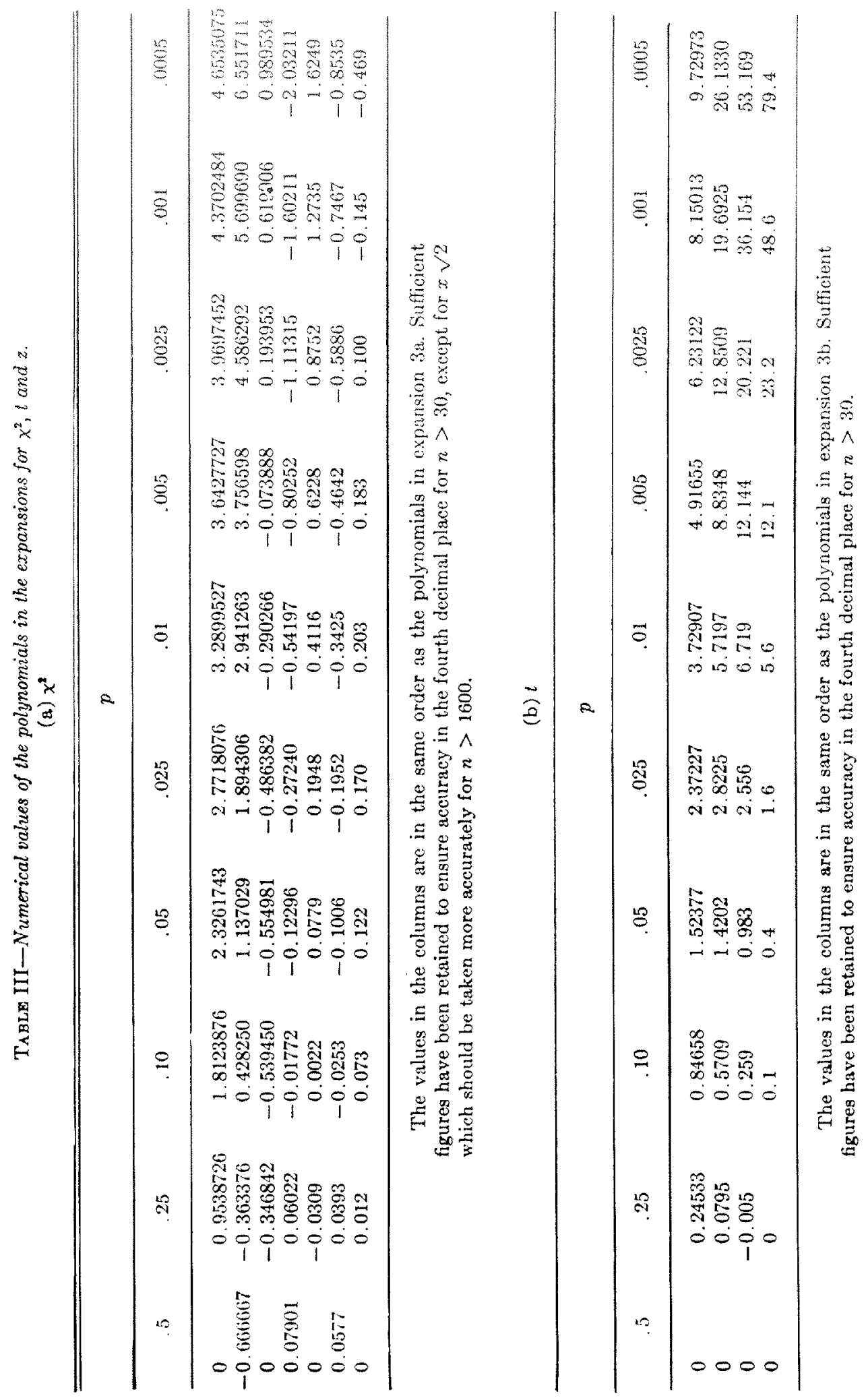




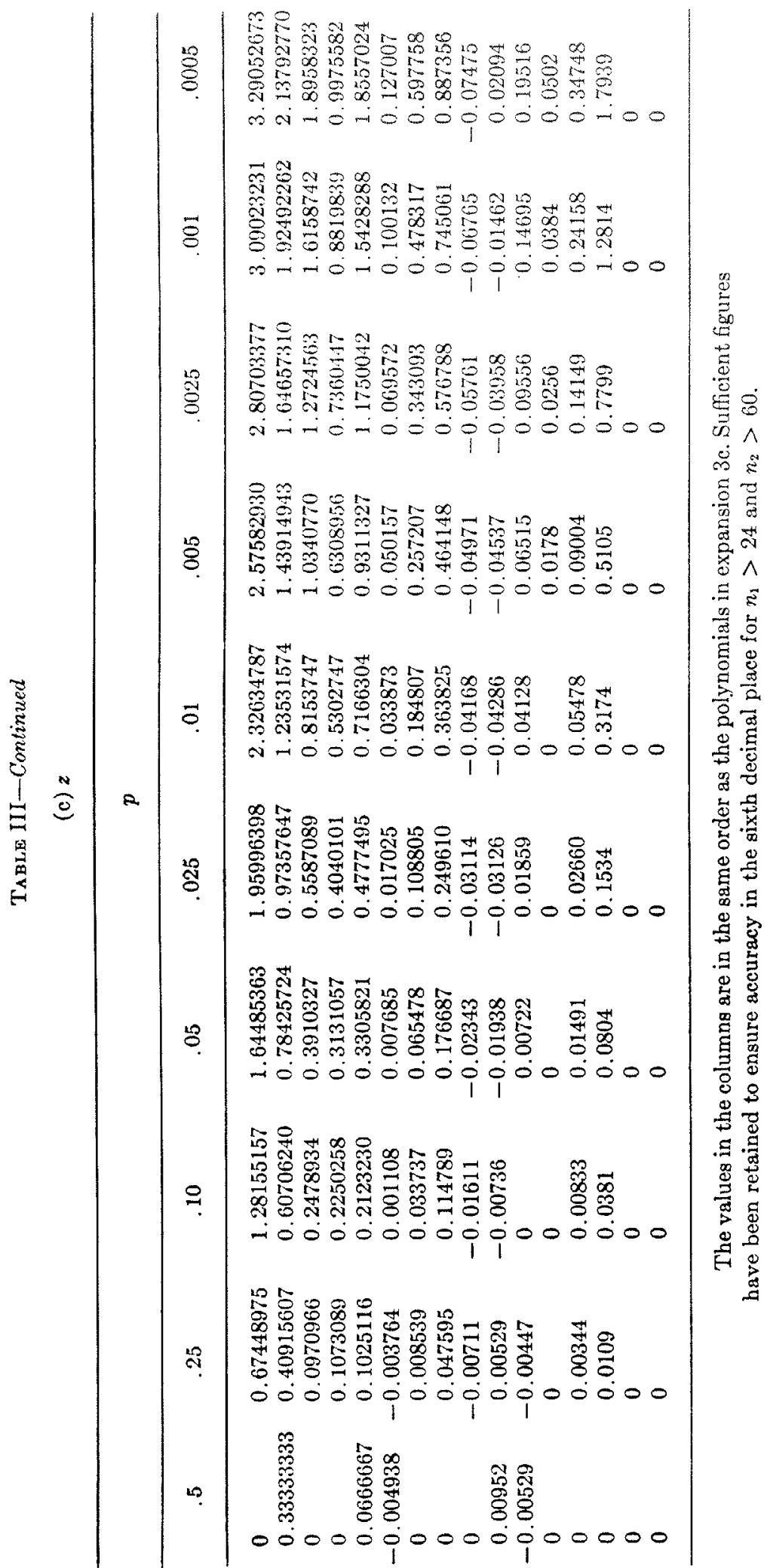


Such as arise in Physies and Astronomy, in which we wish to compare estimates of the value of the same quantity (a) from relatively ample data of low intrinsic accuracy, and (b) from a small series of observations of relatively high precision. When, as often happens, the estimates of precision of the means obtained in these two ways are of the same order of magnitude, the only satisfactory test is that based on Behrens' solution. The asymptotic expansion is particularly suitable for evaluating the percentiles for this special application. There were thus four manifest advantages of the asymptotic approach to Behrens' integral (Fisher 1941):

(i) a check on Sukhatme's values, obtained by a completely independent method, and applicable at least for the higher values of $n_{1}$ and $n_{2}$,

(ii) greater accuracy than could be obtained for percentiles from Sukhatme's table for values of $n_{1}$ and $n_{2}$ greater than 12 ,

(iii) a wider range of levels of significance in the region to which the asymptotic expansion is applicable,

(iv) the theoretical guidance offered by the algebraic form of the leading terms of the expansion.

(b) The fiducial distribution of the binomial parameter, $p$

When discussing the application of the fiducial argument to discontinuous observations, Fisher (1959) found that the mean of the fiducial distribution of $p$, the parameter of the binomial distribution, for given observational frequencies $a, b$ out of $N$, was

$$
\vec{p}=\frac{a}{N}+\frac{b-a}{2 N^{-3}}-\frac{3(b-a)}{2 N^{3}}+\frac{15(b-a)}{2 N^{4}}-\cdots
$$

if $\chi$ were taken to be normally distributed.

On the other hand, the mean of the Bayesian distribution a posteriori, using the Bayesian probability a priori

$$
\frac{1}{\pi \sqrt{p q}} d p
$$

was

$$
\frac{a+\frac{1}{2}}{N+1}=\frac{a}{N}+\frac{b-a}{2 N^{2}}-\frac{b-a}{2 N^{3}}+\frac{b-a}{2 N^{4}}-\cdots .
$$

Asymptotic agreement between these means appears when allowance is made for the effects of departure from normality in the binomial distribution, which are appreciable in the expression (4).

Direct application of the asymptotic expansion, using the six adjustment terms gave the following expansion for the binomial variate in terms of the normal deviate $x$

$a=p N+x \sqrt{N p q}+\frac{1}{6}(q-p)\left(x^{2}-1\right)$

$$
+\frac{1}{72 \sqrt{N p q}}\left\{-x^{3}+x-p q\left(2 x^{3}-14 x\right)\right\}+\cdots
$$


which, after inversion, gave*

$$
\begin{aligned}
p=\frac{a}{N}-\frac{x \sqrt{a b}}{N^{3 / 2}} & +\frac{(b-a)\left(2 x^{2}+1\right)}{6 N^{2}} \\
& +\left\{\frac{\left(-2 N^{2}+26 a b\right) x^{3}+\left(-7 N^{2}+34 a b\right) x}{72 N^{5 / 2} \sqrt{a b}}\right\}+\cdots
\end{aligned}
$$

whence, substituting its average value for each power of $x$, the mean of the fiducial distribution is

$$
\vec{p}=\frac{a}{N}+\frac{b-a}{2 N^{2}}-\frac{b-a}{2 N^{3}}+\frac{b-a}{2 N^{4}}-\cdots
$$

agreeing so far as the fourth term with (5).

The expansion (6) also provides a ready means for comparing the two distributions with respect to other properties. For example, although the means are in agreement, the asymptotic fiducial distribution has the higher variance.

See also the alternative treatment in Fisher (1957).

\section{(c) Quantilative inherilance}

Panse (1940) has described a statistical technique for the study of quantitative inheritance, in which genetic models, based on data from the $F_{2}$ and $F_{3}$ generations, are used to represent the constitution of particular characters. The statistical consequences in the population, corresponding to these models were assessed, using the cumulant function of the joint distribution of the $F_{2}$ phenotypic value, the mean of the $F_{3}$ progeny, and the genotypic variance of $F_{3}$ progeny. These functions provided the data for expressing an attribute of the $F_{3}$ progeny in.terms of the $F_{2}$ phenotypic values, and thus the effects of selection in the $F_{2}$ phenotype on the mean value of the $F_{3}$ progeny could be determined by integration over the $F_{2}$ distribution. When the intensity of selection was assigned, the limits of integration were calculable from the asymptotic expansion of the deviate.

\section{A Class of Distributions with a Finite Condensation at Zero}

The Poisson Series, a discontinuous distribution of positive integers, is well known to have the simple series of cumulants

$$
\kappa_{r}=m,
$$

for all values of $r$. Correspondingly, the cumulative function is

$$
K=m\left(e^{i \ell}-1\right)
$$

and the characteristic function

$$
M=\exp \left\{m\left(e^{i \iota}-1\right)\right\}
$$

* For the remaining terms see Fisher (1959) 
It is less well known that if, for all values of $r$,

$$
\kappa_{r}=r ! m
$$

or, if a scaling factor be introduced,

$$
\kappa_{r}=r ! a^{r} m
$$

the distribution, derived from

$$
K=\frac{m a i t}{1-a i t}
$$

and

$$
\begin{aligned}
M & =e^{-m} e^{m /(1-a i t)} \\
& =e^{-m} \sum_{0}^{\infty} \frac{m^{n}}{n !}(1-a i t)^{-n}
\end{aligned}
$$

is continuous over the range of positive values, with a finite condensation at zero. For $(1-a i t)^{-\mathbf{n}}$ is the characteristic function of the Eulerian distribution

$$
\frac{1}{(n-1) !}\left(\frac{X}{a}\right)^{n-1} e^{-x / a} \frac{d X}{a}
$$

or of

$$
\chi^{2}=\frac{2 X}{a},
$$

for $2 n$ degrees of freedom, or of the sum of $n$ random variables, each distributed as

$$
e^{-x / a} \frac{d x}{a}, x \geq 0 \text {, a positive }
$$

Hence the distribution is that of the sum of a number of such variables, when the number is distributed in a Poisson Series of parameter $m$. The variate is, therefore, never negative, but is zero with finite frequency

$$
e^{-m} \text {. }
$$

Over the range of positive values, the distribution is continuous, and can be expressed as

$$
\sqrt{\frac{m}{x a}} e^{-m-(x / a)} I_{1}\left(2 \sqrt{\frac{x m}{a}}\right) d x
$$

where $I_{1}$ is a Bessel function, specified by

$$
I_{1}(u)=\frac{1}{2} u+\frac{1}{2^{2} \cdot 4} u^{3}+\frac{1}{2^{2} \cdot 4^{2} \cdot 6} u^{5}+\cdots
$$

The distribution was first recognized (Bennett 1954, Fisher 1954) as characteristic of that of the length of germ plasm still heterogenic at an advanced stage of inbreeding, but its intrinsic incorporation of a finite condensation at 
zero makes it appropriate to a number of natural phenomena, a good illustration being the rainfall of an arid region. A continuous model for rainfall is unsatisfactory for such regions. For many localities it is preferable to use a model ascribing the total rainfall for a given period, for example, a month or a year, to a number of showers, the number being a random sample from a Poisson series with parameter $m$, the rainfall of the showers having positive values only, representable by the Eulerian distribution

$$
\frac{1}{p !} x^{p} e^{-x} d x
$$

where $p$ can be small, as in the previous example where it is actually 0 , but must be $>-1$.

The advantage of this type of distribution, relevant for the purposes of the water engineer, is that there is a finite probability, namely $e^{-m}$, of no rain, whereas a continuous distribution would make this probability zero, contrary to experience.

For the asymptotic expansion, when $m$ is sufficiently large, we may take the exact values for the mean $(m)$ and variance $(2 m)$ and for the measures of non-normality

$$
\begin{array}{ll}
c=\frac{3}{\sqrt{2 m}} & f=\frac{90}{m^{2}} \\
d=\frac{6}{m} & g=\frac{315 \sqrt{2}}{m^{2} \sqrt{m}} \\
e=\frac{15 \sqrt{2}}{m \sqrt{m}} & h=\frac{2520}{m^{3}} .
\end{array}
$$

These yield the six adjustments, to the normal deviate $x$

$$
\begin{array}{ll}
\text { I } \frac{x^{2}-1}{\sqrt{8 m}} & \text { IV }-\frac{4 x^{3}-x}{384 m^{2}} \\
\text { II } \frac{-x}{8 m} & \text { V } \frac{3 x^{4}+2 x^{2}-11}{480 m^{2} \sqrt{2 m}} \\
\text { III } \frac{x^{2}-1}{24 m \sqrt{2 m}} & \text { VI }-\frac{96 x^{5}+164 x^{3}-767 x}{46080 m^{3}} .
\end{array}
$$

The coefficients of Table IV give a rather comprehensive tabulation of the distribution, when $m$ is sufficiently large for the accuracy required, and the levels of significance of interest. These large values of $m$ would be troublesome to use in direct evaluation. For sufficiently small values of $m$, however, the probability that the variate $x$ exceeds any limit $X$ may be evaluated as

$$
P=e^{-m} e^{-x} \sum_{i=0}^{\infty} \frac{X^{i}}{i !} \sum_{i>i} \frac{m^{i}}{j !}
$$

which may be recognized as the probability that a random Poisson variate with parameter $m$ shall exceed a random Poisson variate with parameter $X$. 


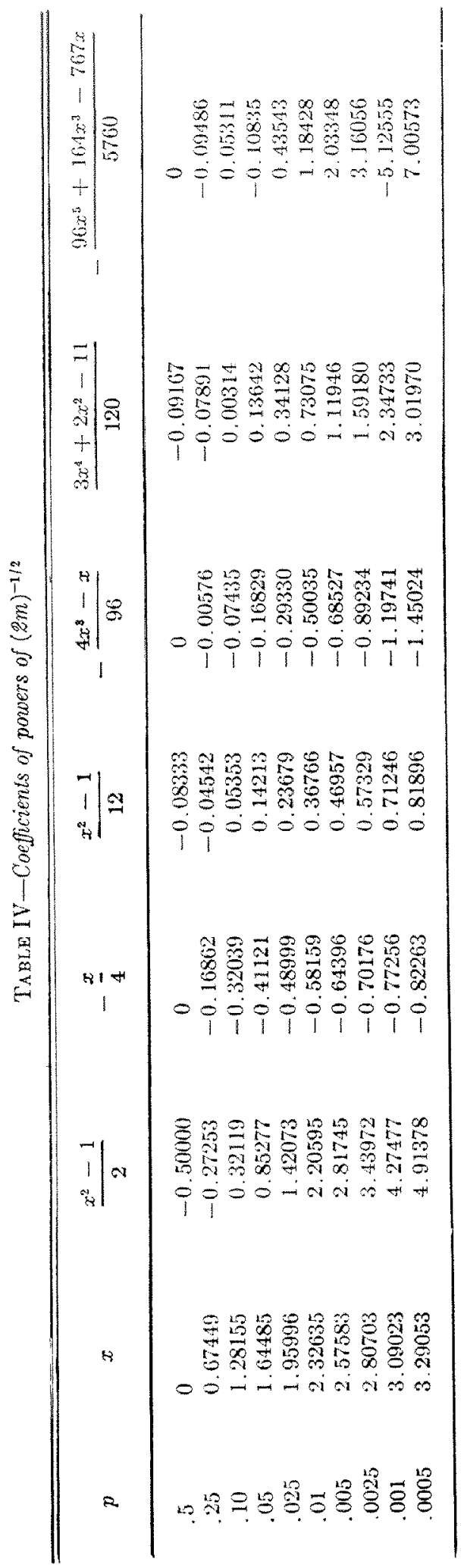




\section{RETERNCES}

J. H. BenNerw (1954). The distribution of heterogeneity upon inbreeding. J. Roy. stat. soc. B, 16:88-99.

E. A. Cornish and R. A. Frsher (1937). Moments and cumulants in the specification of diztributions. Rev. de l'Inst. int. de stat. 5: 307-22.

R. A. Fisher (1926). Expansion of "Student's" integral in powers of $n^{-1}$. Metron 5: 109-12.

R. A. Frsher (1941). The asymptotic approach to Behrens' integral, with further tables for the $d$ test of significance. Ann. eugen. 11: 141-72.

R. A. Fisher (1954). A fuller theory of "junctions" in inbreeding. Heredity 8: 187-97.

R. A. Fisher (1957). The underworld of probability. Sankhya 18: 201-10.

R. A. Frsher (1959). "Statistical Methods and Scientific Inference." 2nd ed. Oliver and Boyd, Edin.

H. Goldberg and H. Levine (1946). Approximate formulas for the percentage points and normalization of $t$ and $\chi^{2}$. Ann. math. statist. 17: 216-25.

N. L. Johnson and B. L. WELCH (1939). Applications of the non-central t-distribution. Biometrika 31 : 362-89.

V. G. PANSE (1940). A statistical study of quantitative inheritance. Ann. eugen. 10: 76-105.

P. V. Sukmatme (1938). On Fisher and Behrens' test of significance for the difference in means of two normal samples. Sankhya 4: 39-48. 